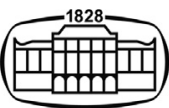

AKADÉMIAI KIADÓ

Journal of Psychedelic Studies

5 (2021) 2, 83-93

DOI:

10.1556/2054.2021.00172

(c) 2021 The Author(s)

\section{ORIGINAL RESEARCH} PAPER

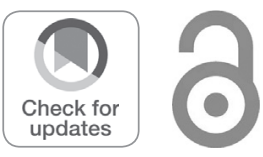

\title{
Lifetime use of psychedelics is associated with better mental health indicators during the COVID-19 pandemic
}

\author{
FEDERICO CAVANNA $^{1,2 *} \odot$, CARLA PALLAVICINI ${ }^{1,2}$, \\ VIRGINIA MILANO ${ }^{3}$, JUAN CUIULE ${ }^{3}$, ROCCO DI TELLA ${ }^{1,3}$, \\ PABLO GONZÁLEZ ${ }^{3}$ and ENZO TAGLIAZUCCHI ${ }^{1,4}$
}

\author{
${ }^{1}$ Departamento de Física, Universidad de Buenos Aires and Instituto de Física de Buenos Aires \\ (IFIBA - CONICET), Pabellón I, Ciudad Universitaria, 1428, CABA, Buenos Aires, Argentina \\ ${ }^{2}$ Fundación para la lucha contra las enfermedades neurológicas de la infancia (FLENI), Montañeses \\ 2325, C1428 CABA, Buenos Aires, Argentina \\ ${ }^{3}$ El Gato y La Caja, Teodoro García 2747, C1426DMU CABA, Buenos Aires, Argentina \\ ${ }^{4}$ Latin American Brain Health Institute (BrainLat), Universidad Adolfo Ibanez, Santiago, Chile
}

Received: February 15, 2021 • Accepted: April 9, 2021

Published online: May 4, 2021

\begin{abstract}
Background and aims: The COVID-19 pandemic and its consequences represent a major challenge to the mental health and well-being of the general population. Building on previous work on the potential long-term benefits of psychedelics, we hypothesized that lifetime use of these drugs could be linked to better mental health indicators in the context of the ongoing pandemic. Methods: Two anonymous online surveys were conducted between April and June 2020, including questions about lifetime experience with psychedelics and other psychoactive drugs, and psychometric scales designed to measure personality traits, anxiety, negative, and positive affect, well-being, and resilience. Principal component analysis was applied to divide the sample into groups of subjects based on their drug use reports. Results: Five thousand six hundred eighteen participants ( $29.15 \pm 0.12$ years, $71.97 \%$ female) completed both surveys and met the inclusion criteria, with $32.43 \%$ of the sample reporting at least one use of a psychedelic drug. Preliminary analyses showed that certain psychedelics were linked to improved mental health indicators, while other psychoactive drugs exhibited the opposite behavior. Lifetime psychedelic use was linked to increased openness and decreased conscientiousness, and to higher scores of positive affect. The reported number of past psychedelic experiences predicted higher scores of the secondary personality trait beta factor, which has been interpreted as a measure of plasticity. No significant associations between lifetime use of psychedelics and indicators of impaired mental health were observed. Conclusion: We did not find evidence of an association between lifetime use of psychedelics and poor mental health indicators. Conversely, experience with psychedelic drugs was linked to increased positive affect and to personality traits that favor resilience and stability in the light of the ongoing crisis.
\end{abstract}

\section{KEYWORDS}

psychedelics, mental health, resilience, well-being, personality traits, COVID-19

\section{INTRODUCTION}

*Corresponding author. E-mail: f.cavanna@gmail.com

Psychedelic drugs are found in nature in multiple species of plants, fungi and animals (Rätsch, 2005). The use of certain compounds (i.e., mescaline, psilocybin, N,N-dimethyltryptamine [DMT]) has been documented at least for centuries, mainly in association with medicinal, religious, and other ceremonial practices (e.g., divination) (Escohotado, 2002). The molecular structure of classic psychedelics resembles that of serotonin (an endogenous 
neurotransmitter), which enables their pharmacological action as $2 \mathrm{~A}$ serotonin receptors $\left(5-\mathrm{HT}_{2 \mathrm{~A}}\right.$ ) agonists (Nichols, 2016). At the phenomenological, cognitive and behavioral levels, $5-\mathrm{HT}_{2 \mathrm{~A}}$ agonism is linked to a wide range of effects that alter the conscious experience of the user, such as modifications in the perception of the environment and the self, sedation, stimulation, as well as changes in mood, prosocial behavior, cognitive flexibility, and creativity, among others (Jungaberle et al., 2018; Nichols, 2016; Palamar \& Acosta, 2020; Preller \& Vollwenweider, 2016; Schmid et al., 2015). These effects attracted the attention of mainstream science during the 1950 s, leading to several investigations in healthy and clinical populations (Dyck \& Farrell, 2018). However, the widespread availability of psychedelic drugs (mainly LSD) during the 1950s and 1960s, combined with a complex social and political landscape, led to the classification of several psychedelics as Schedule 1 drugs, which effectively shut down most research on these substances and their potential clinical uses (Rucker, Iliff, \& Nutt, 2018).

The prohibition on psychedelic drugs was partially justified by concerns regarding potential long-term negative effects on mental health (Lee \& Shlain, 1992). However, subsequent studies failed to establish a link between lifetime use of psychedelic drugs and increased rate of mental health issues. Krebs and Johansen analyzed data from 130,152 randomly selected individuals, of which 21,967 reported at least one experience with psychedelic drugs (Krebs \& Johansen, 2013). Their study failed to detect significant associations between psychedelic use and several markers of impaired mental health, including serious psychological distress, inpatient or outpatient mental health treatment, and symptoms of nine psychiatric disorders. A follow-up study by the same authors found the same results after adjusting for sociodemographics, the use of other drugs, and childhood depression (Johansen \& Krebs, 2015). Converging results have been published for controlled laboratory studies (Nichols, 2016). Assessments by interdisciplinary panels of experts consistently ranked psychedelics as some of the least harmful recreational drugs, with safety profiles substantially better than those of widely available and consumed drugs, such as alcohol and tobacco (Nutt, King, \& Phillips, 2010).

In recent years, the scientific and clinical interest in psychedelics has increased considerably as a consequence of a new wave of research demonstrating their potential in neuroscience, psychiatry, and as adjuncts for psychotherapy (Johnson \& Griffiths, 2017; Kleber, 2016; Nichols \& Hendricks, 2020; Nutt \& Carhart-Harris, 2020; Reiff et al., 2020). One of the major promises of psychedelics as therapeutic agents is their capacity to induce long-term psychological and behavioral changes after a single session (Bouso, dos Santos, Alcázar-Córcoles, \& Hallak, 2018; Erritzoe et al., 2018, 2019; Lebedev et al., 2016; MacLean, Johnson, \& Griffiths, 2011), which highlights their enormous potential in the treatment of certain mood disorders and addictive behaviors (Bogenschutz et al., 2015; Johnson, GarciaRomeu, Cosimano, \& Griffiths, 2014; Johnson, GarciaRomeu, \& Griffiths, 2017). Crucially, these effects appear to be mediated by the nature of the induced psychedelic experience, with reports of mystical-type experiences being associated with better outcomes (Garcia-Romeu, Griffiths, \& Johnson, 2014; Griffiths et al., 2018; Johnson, Hendricks, Barrett, \& Griffiths, 2019). While these effects have been shown for specific clinical populations, it could be hypothesized that psychedelic use might lead to long-term mental health benefits in healthy individuals. These benefits might remain unnoticed until the individual is challenged by adverse personal or social circumstances.

We investigated the relationship between lifetime psychedelic use and multiple mental health indicators in the context of the COVID-19 pandemic, an adverse event of worldwide scope. COVID-19 is a contagious respiratory and vascular disease caused by acute respiratory syndrome coronavirus 2 (SARS-CoV-2). The rapid spread of COVID-19 during early 2020 precipitated drastic lockdown measures in several countries, which in turn impacted negatively in the mental health of the population (Pfefferbaum \& North, 2020); in particular, this study was conducted in Argentina, where one of the longest lockdowns was declared between March and November. Our first main objective was to determine differences in mental health indicators (anxiety, positive and negative affect, well-being, resilience) between individuals who reported past psychedelic use, and those who declared their past use of other psychoactive drugs. Our second objective was to investigate the potential impact of lifetime psychedelic use on personality traits, and how this potential impact related to different mental health indicators.

\section{MATERIALS AND METHODS}

\section{Survey}

An anonymous Internet-based survey with two parts was conducted between April 2020 and June 2020, i.e., between one and three months after a severe lockdown was declared in most of Argentina's provinces. Recruitment advertisements were shared via social media web pages (e.g., Facebook, Instagram, and Twitter). The first part of the survey was promoted as a questionnaire to understand the relationship between psychoactive drug use and mental health during the COVID-19 pandemic, while the second part was promoted as a questionnaire to determine personality traits. The survey was presented in Spanish and each of its parts took approximately 20 minutes to complete. The inclusion criteria were providing online informed consent, being Argentinian, older than 18 years and declaring male or female gender identity. Subjects received no compensation for their participation.

\section{Ethical approval}

The procedure was included within a larger protocol for online experimentation approved by the ethics committee of Centro de Educación Médica e Investigaciones Clínicas Norberto Quirno. All participants gave their informed consent before their participation in the present study. 


\section{Questionnaires and scales}

The full survey is available online (https://investigacion. elgatoylacaja.com/concienciaysustancia/ and https:// investigacion.elgatoylacaja.com/personalidad/). The first questionnaire collected sociodemographic variables (age, gender, and nationality), variables related to past use of psychoactive compounds (drugs that were consumed by the user and their frequency of use - for a brief description of the drugs included in the survey see the supplementary material), and the STAI, PANAS, BIEPS, and RS scales. The second part of the survey assessed personality via the BFI questionnaire. Both questionnaires were independent from each other.

State-Trait Anxiety Inventory (STAI): a commonly used scale which measures state anxiety (which refers to a situational anxiety of a temporary nature) and trait anxiety (which designates a stable trait linked to personal characteristics) (Spielberger, Gorsuch, Lushene, \& Inc, 1983). The instrument comprises 40 items and is based on a 4-point Likert scale, ranging from "Almost Never" to "Almost Always".

Positive and Negative Affect Schedule (PANAS): a psychometric scale that has been widely used to measure both proposed dimensions of affect, positive and negative (Watson, Clark, \& Tellegen, 1988). The instrument consists of 20 affirmations based on a 5-point Likert scale, ranging from "Not at all" to "Very much".

Psychological Well-being Scale (BIEPS): a scale used to measure eudaimonic well-being in adults (including dimensions of acceptance, perception of control, social ties, and autonomy and projects) (Casullo \& Brenlla, 2002). It consists of 13 questions based on a 3-point Likert scale, ranging from "Disagree" to "Agree".

Resilience Scale (RS): a questionnaire aiming to evaluate Resilience through the subscales of self-reliance, purpose capacity and life meaning and cognitive avoidance (Wagnild \& Young, 1993). In this study a local adaptation was used (Rodríguez et al., 2009) which consists of 21 items based on a 5-point Likert scale, ranging from "Fully agree" to "Fully disagree".

Big Five Inventory (BFI): an inventory assessing five dimensions of personality: neuroticism, extraversion, openness to experience, agreeableness, and conscientiousness (Benet-Martinez \& John, 1998). The BFI questionnaire consists of 44 items based on a 5-point Likert scale, ranging from "Fully disagree" to "Fully agree".

\section{Data processing and analysis}

Data from the two questionnaires was merged according to a unique identifier. Due to the scope of the ethics approval, and because the local adaptation of psychometric scales is limited to male and female Argentinian adults, only participants older than 18 years old, residents of Argentina, and with male or female gender identity were retained for subsequent analysis.

Psychometric scales (i.e., STAI, PANAS, BIEPS, RS, BFI) were scored using the subject's answers, gender, and age to obtain a value representing the results compared to values previously obtained and tabulated for the local population within the same gender and age group, according to the procedure specified in each psychometric manual. Next, all values were converted to a uniform scale between 0 and 10 (i.e., $z$-score) in order to facilitate comparison.

Data analysis was performed using Python 3.7. Descriptive statistics (e.g., means, standard deviations, and standard errors from the mean [SEM]) were used to characterize the scale and subscale scores of the survey. Pearson's linear correlation coefficient was computed between all pairs of scales and subscales to assess whether the different dimensions of our dataset were related as expected (e.g., positive correlations between the dimensions related to good mental health). To reduce the dimensionality of the data and thus the number of independent statistical tests, and to avoid the issue of non-independent samples, we first conducted a principal component analysis (PCA) of the data based on computing the singular value decomposition of the centered data matrix. The rows of the data matrix corresponded to individual participants, and its columns contained binary values indicating whether the participants reported lifetime use of each drug, i.e., one drug per column. After grouping the drug use variables into interpretable principal components, we applied analysis of variance (ANOVA) followed by Student's $t$-tests to determine statistically significant differences between groups of users assigned to the different principal components. Bonferroni correction for multiple comparisons was applied whenever stated in the description of the results. We note that PCA allowed us to group drugs whose reported lifetime uses were highly correlated in a data-driven way, thus avoiding the issue of non-independent samples (i.e., subjects who consumed several drugs in the past) without introducing ad-hoc groups.

\section{RESULTS}

During the data collection window (April 2020-June 2020), 11,365 individuals answered the first part of the survey (demographics, drug use questionnaire, STAI, PANAS, BIEPS, RS) and 157,101 answered the second part (BFI questionnaire). Although both parts of the survey were promoted in the same way, the second part achieved a much higher level of dissemination in social media than the first part, which led to the difference in the number of responses. Of all the subjects, 10,722 answered both parts of the survey. Of these, 5,104 were excluded as they failed to meet the inclusion criteria presented in the "Materials and methods" section. Thus, the final sample consisted of 5.618 participants who were $29.15( \pm 0.12)$ years old and $71.97 \%$ female. $32.43 \%$ of the total sample reported at least one use of a psychedelic drug. All data is freely available at https://doi. org/10.5281/zenodo.4324025.

The demographic information is summarized in Table 1. Note that percentages in the first row of Table 1 do not add to $100 \%$, since reported uses of different drugs are not exclusive (e.g., one participant could have reported past uses 
Table 1. The number of users, their gender identity and age corresponding to the subset of 5,618 participants who met the inclusion criteria (i.e., Argentinian residents older than 18 years with male or female gender identity) classified by drug, by total number of drug users and non-users, and by total sample

\begin{tabular}{lcccccccccc}
\hline & LSD or & & & 5 meo- & San & Bufo & Total & Total & Total \\
users & Pedro & Alvarius & non-users & sample \\
\hline$\%(n)$ & $9.9 \%(556)$ & $30.24 \%$ & $1.25 \%(70)$ & $2.79 \%$ & $0.57 \%$ & $1.51 \%$ & $0.12 \%$ & $32.43 \%$ & $67.57 \%$ & $100 \%$ \\
& & $(1,699)$ & & $(157)$ & $(32)$ & $(85)$ & $(7)$ & $(1,822)$ & $(3,796)$ & $(5,618)$ \\
Female (\%) & $52.70 \%$ & $63.21 \%$ & $62.86 \%$ & $61.15 \%$ & $75.00 \%$ & $54.12 \%$ & $42.86 \%$ & $63.17 \%$ & $76.19 \%$ & $71.97 \%$ \\
Male (\%) & $47.30 \%$ & $36.79 \%$ & $37.14 \%$ & $38.85 \%$ & $25.00 \%$ & $45.88 \%$ & $57.14 \%$ & $36.83 \%$ & $23.81 \%$ & $28.03 \%$ \\
Age (mean \pm & $28.89 \pm$ & $27.41 \pm$ & $33.81 \pm$ & $27.54 \pm$ & $29.50 \pm$ & $31.52 \pm$ & $38.00 \pm$ & $27.66 \pm$ & $29.86 \pm$ & $29.15 \pm$ \\
SEM) & 0.28 & 0.16 & 1.00 & 0.47 & 1.12 & 0.82 & 4.42 & 0.16 & 0.16 & 0.12 \\
\hline
\end{tabular}

of different drugs). Similarly, Table 2 summarizes demographic information for each PCA group and shows the results of each psychometric subscale included in the survey.

Figure 1 summarizes the results of the BFI questionnaire (panel A), and of the STAI, PANAS, BIEPS, and RS scales (panel B). The latter were first transformed to Z-scores, so that scores below/above zero indicate values that are below/ above the regional averages (these averages are the result of the statistical analysis carried out when adapting a psychometric scale in a given population). We observed that positive affect (PA), well-being (W), and resilience (R) scores were considerably below zero, which we can interpret as a potential effect of the COVID-19 pandemic and its consequences. Figure $1 \mathrm{D}$ and $\mathrm{E}$ shows the same information as in panels $\mathrm{A}$

Table 2. Survey results grouped by principal component scores. Psychometric scales and subscales results (STAI, PANAS, BIEPS, RS, BFI) are presented as mean \pm standard error

\begin{tabular}{|c|c|c|c|c|}
\hline & \multicolumn{4}{|c|}{ PCA components } \\
\hline & Recreational & Entheogen & Prescribed & Legal/Non-users \\
\hline \multicolumn{5}{|l|}{ Demographics } \\
\hline$\%(n)$ & $23.63(1,328)$ & $3.06(172)$ & $12.99(730)$ & $48.94(2,750)$ \\
\hline Sample percentage (\%) & 23.64 & 3.06 & 12.99 & 48.95 \\
\hline Female (\%) & 61.22 & 58.72 & 84.93 & 74.76 \\
\hline Male (\%) & 38.78 & 41.28 & 15.07 & 25.24 \\
\hline Age $($ mean \pm sem $)$ & $27.21 \pm 0.17$ & $29.84 \pm 0.61$ & $32.59 \pm 0.41$ & $29.16 \pm 0.18$ \\
\hline Psych. assistance (\%) & 59.64 & 56.98 & 82.33 & 51.96 \\
\hline Hospitalization (\%) & 0.15 & 1.16 & 3.29 & 0.25 \\
\hline Medication (\%) & 0.53 & 0.00 & 24.25 & 0.25 \\
\hline \multicolumn{5}{|l|}{ STAI } \\
\hline State anxiety & $24.62 \pm 0.29$ & $23.39 \pm 0.85$ & $29.08 \pm 0.45$ & $25.32 \pm 0.22$ \\
\hline Trait anxiety & $25.25 \pm 0.29$ & $24.51 \pm 0.85$ & $29.74 \pm 0.45$ & $25.21 \pm 0.22$ \\
\hline \multicolumn{5}{|l|}{ PANAS } \\
\hline Negative affect & $21.64 \pm 0.20$ & $21.23 \pm 0.56$ & $24.57 \pm 0.30$ & $21.43 \pm 0.15$ \\
\hline Positive affect & $29.15 \pm 0.21$ & $30.90 \pm 0.61$ & $28.14 \pm 0.29$ & $29.33 \pm 0.15$ \\
\hline \multicolumn{5}{|l|}{ BIEPS } \\
\hline Acceptance & $7.62 \pm 0.04$ & $7.72 \pm 0.10$ & $7.38 \pm 0.06$ & $7.64 \pm 0.03$ \\
\hline Autonomy & $6.62 \pm 0.04$ & $6.90 \pm 0.12$ & $6.51 \pm 0.06$ & $6.60 \pm 0.03$ \\
\hline Social ties & $8.18 \pm 0.03$ & $8.17 \pm 0.09$ & $7.88 \pm 0.05$ & $8.06 \pm 0.02$ \\
\hline Goals & $10.02 \pm 0.05$ & $10.24 \pm 0.13$ & $9.73 \pm 0.07$ & $10.17 \pm 0.03$ \\
\hline Total well-being & $32.45 \pm 0.11$ & $33.02 \pm 0.30$ & $31.50 \pm 0.17$ & $32.47 \pm 0.08$ \\
\hline \multicolumn{5}{|l|}{ RS } \\
\hline Self-reliance & $57.58 \pm 0.24$ & $58.35 \pm 0.68$ & $55.65 \pm 0.36$ & $57.71 \pm 0.18$ \\
\hline Meaning & $22.76 \pm 0.13$ & $23.18 \pm 0.37$ & $22.02 \pm 0.19$ & $23.48 \pm 0.09$ \\
\hline Cognitive avoidance & $17.99 \pm 0.12$ & $17.78 \pm 0.35$ & $16.65 \pm 0.18$ & $18.22 \pm 0.09$ \\
\hline Total resilience & $98.32 \pm 0.40$ & $99.31 \pm 1.12$ & $94.33 \pm 0.61$ & $99.41 \pm 0.30$ \\
\hline \multicolumn{5}{|l|}{ BFI } \\
\hline Extraversion & $26.36 \pm 0.16$ & $26.42 \pm 0.45$ & $25.40 \pm 0.23$ & $25.57 \pm 0.12$ \\
\hline Agreeableness & $32.88 \pm 0.14$ & $32.35 \pm 0.36$ & $32.61 \pm 0.19$ & $33.01 \pm 0.10$ \\
\hline Conscientiousness & $29.49 \pm 0.17$ & $29.49 \pm 0.51$ & $30.64 \pm 0.25$ & $31.08 \pm 0.13$ \\
\hline Neuroticism & $24.69 \pm 0.18$ & $24.41 \pm 0.47$ & $28.26 \pm 0.23$ & $25.16 \pm 0.13$ \\
\hline Openness & $39.09 \pm 0.17$ & $40.64 \pm 0.45$ & $38.34 \pm 0.24$ & $37.70 \pm 0.13$ \\
\hline Alpha factor & $77.69 \pm 0.33$ & $77.44 \pm 0.93$ & $74.98 \pm 0.48$ & $78.93 \pm 0.24$ \\
\hline Beta factor & $65.44 \pm 0.26$ & $67.06 \pm 0.70$ & $63.74 \pm 0.38$ & $63.27 \pm 0.19$ \\
\hline
\end{tabular}


and $\mathrm{B}$, but segmented according to the reported number of psychedelic drug uses. The clearest trends can be observed in panel D for the BFI scores, with the openness (O) and extraversion (E) traits increasing as a function of reported uses, and conscientiousness (C) showing the opposite behavior. Figure $1 \mathrm{C}$ presents all pairwise correlations between subscales with $|R|>0.3$. The openness and agreeableness dimensions of the BFI questionnaire were not correlated with any of the other scales, while expected positive correlations between state/trait anxiety (SA/TA), negative affect (NA), and neuroticism were observed. Resilience and well-being were positively correlated (and negatively correlated with state/trait anxiety, negative affect and neuroticism), and also presented positive correlations with positive affect, extroversion, and conscientiousness. Alpha factor (AF) - which is conceptualized as a combination of agreeableness, conscientiousness and the inverse of neuroticism-showed positive correlations with traits and states associated with healthy individuals (i.e., PA, $\mathrm{W}, \mathrm{R}, \mathrm{A}, \mathrm{C})$ and negative correlations with states and traits associated with a loss of functionality (i.e., SA, TA, NA, N), whereas Beta factor (BF) - which is obtained as a combination of extraversion and openness-, showed only positive correlations with traits and states associated with healthy individuals (i.e., W, R, E, O).

We then performed a preliminary analysis by grouping all scores from users who reported experience with each drug and comparing those scores with the rest of the sample using Student's $t$-tests. Results are shown in Fig. 2. A double dissociation effect is apparent: some psychedelic drugs (mainly psilocybin mushrooms but also LSD and, to a lesser degree, ayahuasca) were associated with lower scores of dimensions linked to mental health impairment (state/trait anxiety, negative affect) and with higher scores of dimensions linked to well-being and resilience (mainly positive affect, autonomy, social ties, and well-being); conversely, other drugs (including prescription drugs, caffeine, alcohol, tobacco, and cannabis) were linked to higher scores of dimensions linked to mental health impairment, and lower scores of dimensions linked to well-being and resilience. Within this last group of non-psychedelic drugs, MDMA showed different results compared to the rest, being associated with higher scores of the social ties dimension of the BIEPS.

We note that this analysis is preliminary since it does not take into account the possibility of a single participant declaring past experience with multiple drugs. To overcome this limitation, we applied PCA to divide the sample into groups of subjects with different drug use profiles. Each group was created based on the scores along three principal components: LSD, MDMA, psilocybin mushrooms, and dissociative drugs ("recreational", i.e., drugs that are frequently consumed in a recreational setting), DMT, ayahuasca, San Pedro (Echinopsis pachanoi), bufo alvarius (Incilius alvarius), and 5-MeO-DMT ("entheogen", i.e., psychedelic compounds that are frequently consumed in religious or ceremonial settings), and antidepressants, antipsychotics, and sedatives ("prescription"). The resulting components are shown in Fig. 3A. To ensure that these groups were mutually exclusive, participants with high scores in the "entheogen" and "recreational" components were only included in the first, e.g., a user with extensive lifetime use of LSD and ayahuasca would only be included in the "entheogen" group. This reflects the observation that most users with lifetime use of drugs in the "entheogen" component also reported experiences with drugs in the "recreational" component, but not vice versa. Subjects presenting high scores for drugs in the "prescription" component were included in the corresponding group, regardless of their scores in the other two components. Finally, we created a group for participants who only declared past experiences with legal drugs, such as caffeine, alcohol and tobacco ("legal/non-users"). $11.36 \%$ of the sample did not clearly fit in any group based on the PCA scores, and hence was discarded from subsequent analysis.
A. BFI trait scores
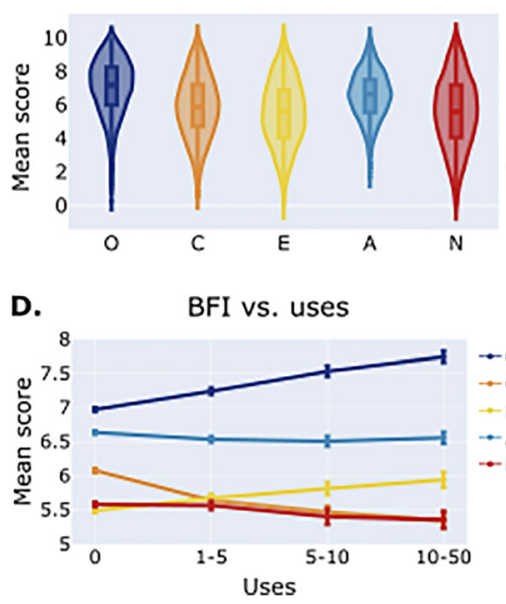

B. Scales trait scores
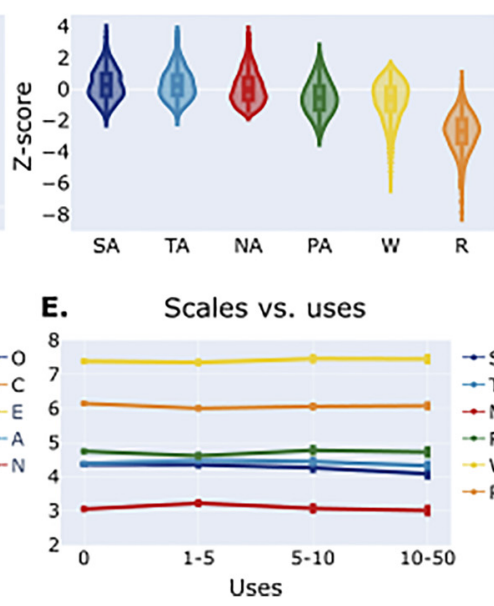

C.
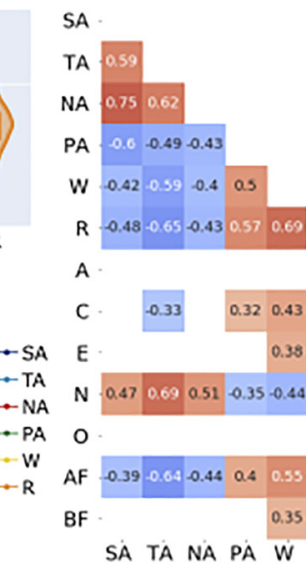

Correlations

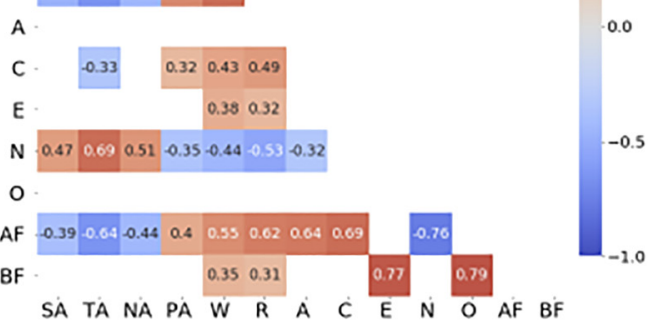

Fig. 1. Summary of the BFI questionnaire (panel A), the STAI, PANAS, BIEPS and RS scales (panel B), both as a function of the reported number of psychedelic drug uses (panels $\mathrm{D}$ and $\mathrm{E}$ ), and all pairwise correlations between subscales with $|R|>0.3$. Abbreviations: $\mathrm{O}$ (Openness), C (Conscientiousness), E (Extraversion), A (Agreeability), N (Neuroticism), SA (State anxiety), TA (Trait anxiety), NA (Negative affect), PA (Positive affect), W (Well-being), R (Resilience), AF (Alpha factor), BF (Beta factor) 


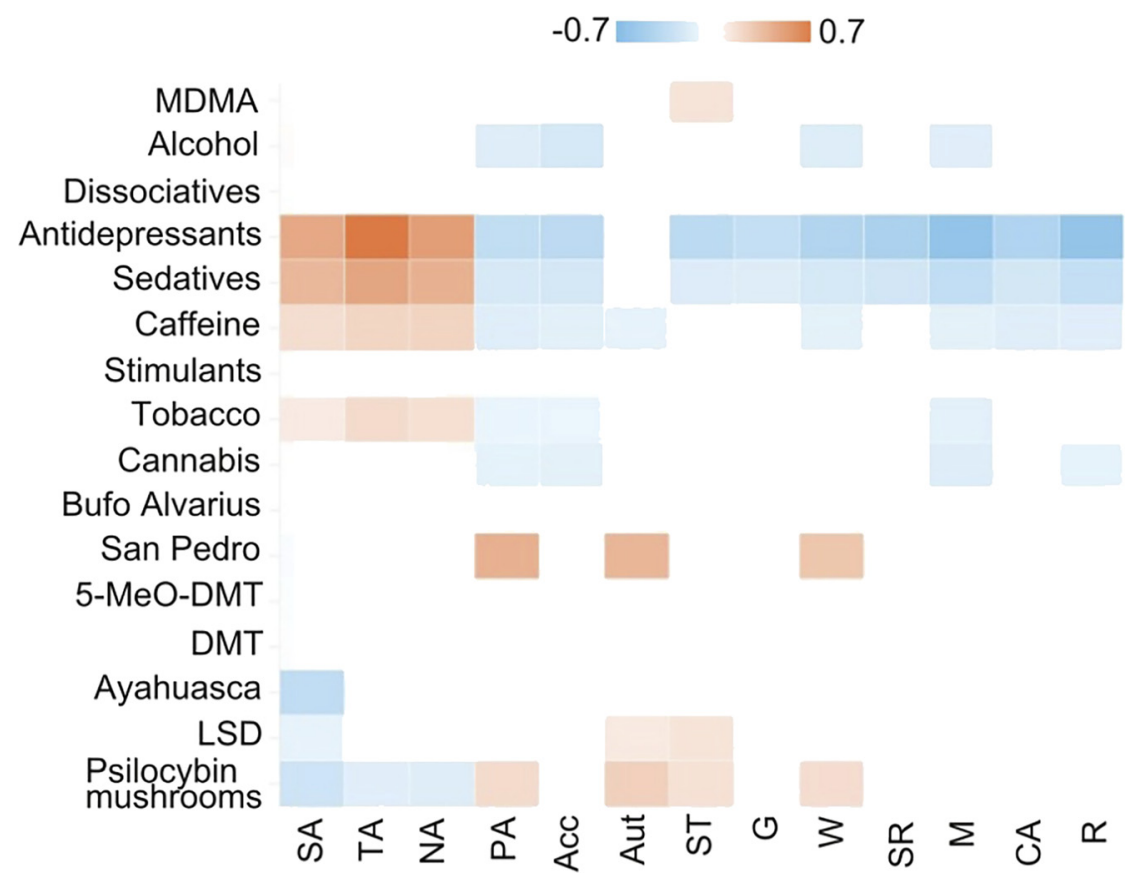

Fig. 2. Preliminary analysis comparing questionnaire scores corresponding to individual drugs vs. all others. Each entry in the matrix corresponds to the effect size (Cohen's d) for the comparison of the score (columns) for the drug (rows) vs. all other drugs grouped together. Only significant entries are shown $(P<0.05$, Bonferroni corrected for $16 \times 13=208$ comparisons). Abbreviations: SA (State anxiety), TA (Trait anxiety), NA (Negative affect), PA (Positive affect), Acc (Acceptance), Aut (Autonomy), ST (Social ties), G (Goals), W (Well-being), SR (Self-reliance), M (Meaning), CA (Cognitive avoidance), R (Resilience)

A.

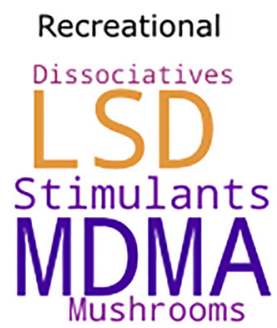

B.

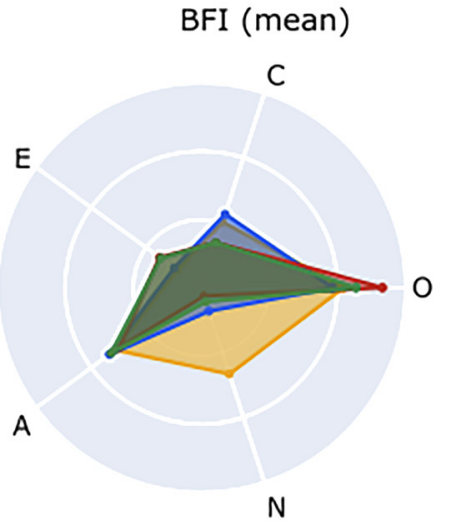

Entheogen

Bufo Alvarius

DMT 5-meo-DMT

Ayahuasca

San Pedro

Scales (mean)

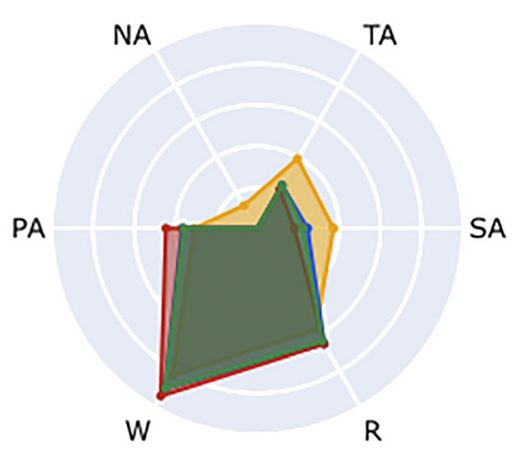

Prescription

Antipsychotics

Antidepressants

Sedatives

Fig. 3. Principal components. A) Word clouds representing the drugs included in the different groups, with word size indicative of the weight of the drug in the corresponding principal component. B) Radar plots showing BFI scores (left), psychometric scale scores (middle) and their subscales (right) for participants belonging to each group, plus a group of participants who only declared past use of legal drugs (e.g., caffeine, alcohol, tobacco). Abbreviations: O (Openness), C (Conscientiousness), E (Extraversion), A (Agreeability), N (Neuroticism), SA (State anxiety), TA (Trait anxiety), NA (Negative affect), PA (Positive affect), W (Well-being), R (Resilience), ACC (Acceptance), AUT (Autonomy), ST (Social ties), G (Goals), SR (Self-reliance), M (Meaning), CA (Cognitive avoidance) 
We first applied an ANOVA test to find a significant effect ( $P<0.05$, Bonferroni corrected for 4 comparisons) of user group in all BFI scores and psychometric scales except for the agreeableness personality trait $(P=0.121)$.

We applied pairwise Student's $t$-test to determine whether the variables plotted in Fig. 3B presented significant differences between the four groups of participants. The results of this analysis are shown in Fig. 4 (state/trait anxiety, BFI, PANAS, BIEPS, and RS) and Fig. 5 (alpha and beta factors). Figure $5 \mathrm{C}$ also presents the second order personality factors alpha and beta as a function of the reported number of lifetime psychedelic experiences.

The "recreational" group presented higher extraversion and lower conscientiousness scores than the group of nonusers, while both "recreational" and "entheogens" resulted in increased openness scores, as expected from previous studies. Also as expected, neuroticism was increased in the "prescription" group relative to all others. No differences were found for the agreeableness personality dimension. Subjects in the "entheogen" group presented higher openness than those in the "recreational" group (Fig. 4A). All measures positively associated with mental health were significantly lower in the "prescription" group; also, we observed higher positive affect in the "entheogen" group compared to all others (Fig. 4B). Finally, the alpha factor was lower for the "prescription" group compared to all others (Fig. 5A), and the beta factor was higher for the "recreational" and "entheogen" groups compared to the other two groups (Fig. 5B). Also, the beta factor increased with the reported number of lifetime psychedelic experiences (Fig. 5C).

\section{A. BFI trait mean scores}
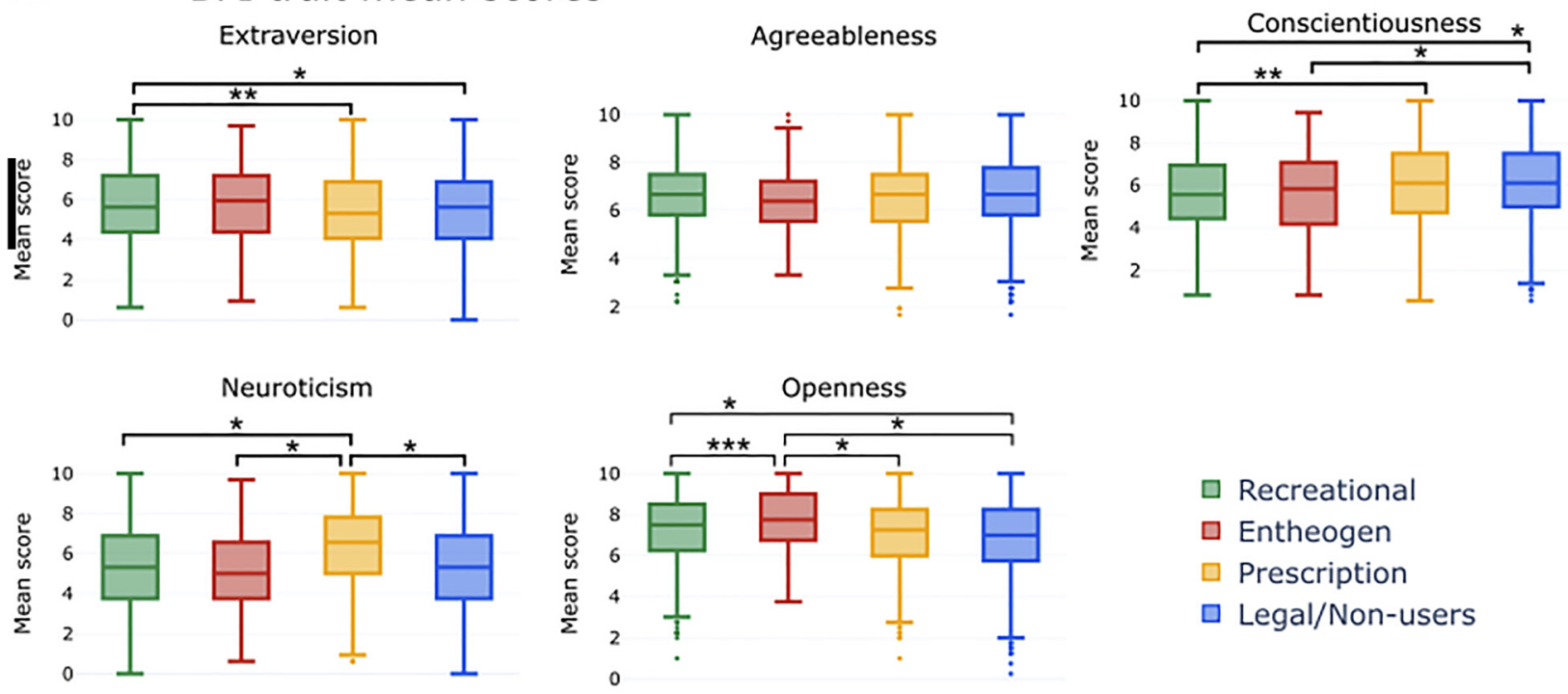

\section{B. Scales mean z-scores}
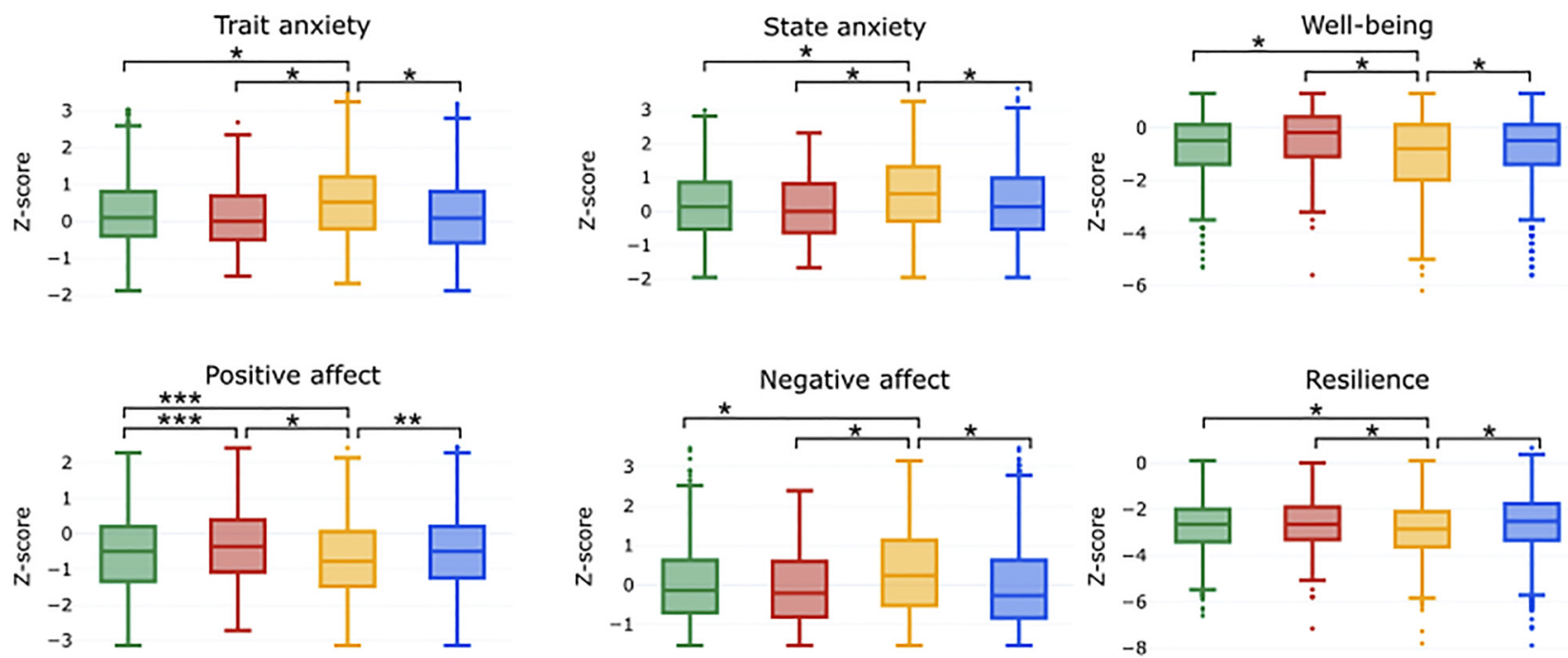

Fig. 4. Comparison of psychometric scales and subscales between groups of participants. A) Comparison of BFI dimensions. B) Comparison of trait/state anxiety, positive and negative affect, well-being and resilience. ${ }^{\star} P<0.05$ (Bonferroni corrected for 4 comparisons), ${ }^{* *} P<0.01$, ${ }^{* * *} P<0.05$ 
A.

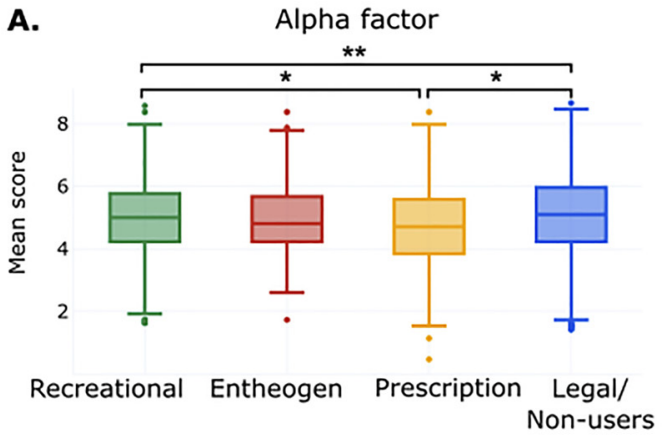

B.

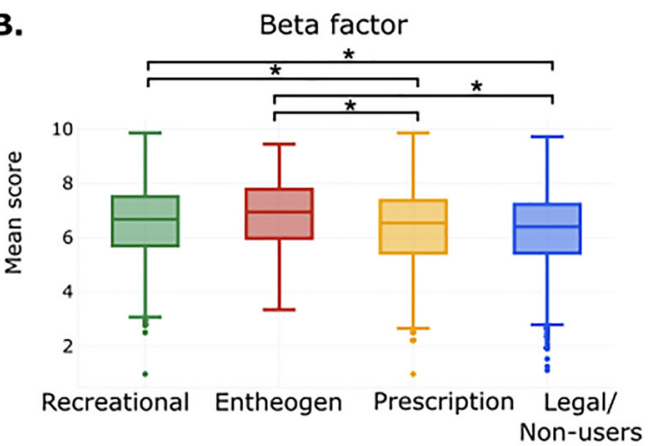

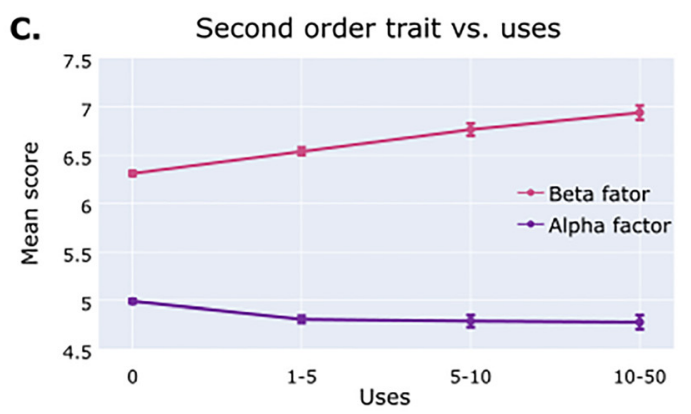

Fig. 5. Comparison of second order personality traits between groups of participants. A) Comparison of alpha factor scores. B) Comparison of beta factor scores. C) Alpha and beta factor vs. reported number of lifetime psychedelic experiences. ${ }^{\star} P<0.05$ (Bonferroni corrected for 4 comparisons), ${ }^{* *} P<0.01,{ }^{* * *} P<0.05$

\section{DISCUSSION}

The current study investigated the relationship between lifetime use of psychoactive drugs and reported levels of anxiety, positive and negative affect, well-being, resilience, and personality traits. We focused our analysis on the possibility that psychedelic drugs could confer sustained benefits, which are manifested as better mental health indicators during the ongoing COVID-19 pandemic.

Concerning the general mental health status of the sample, after scoring positive and negative scales and transforming them to $z$-scores we compared the results to the regional average and observed reduced values, particularly in resilience $(-2.74)$, but also in well-being $(-0.74)$ and PANAS positive affect $(-0.54)$, and higher values in STAI state anxiety (0.3), STAI trait anxiety (0.28), and PANAS negative affect (0.06) (Fig. 4, panel B), in all cases compared to the normative values corresponding to the age- and gender-adjusted regional average. We expected an effect on mental health indicators as a consequence of the social isolation and uncertainty associated with the COVID-19 outbreak. This result is in line with preliminary reports showing serious consequences in the mental health of the general population during the pandemic (Brooks et al., 2020; Gallagher et al., 2020; Pfefferbaum \& North, 2020; Zacher \& Rudolph, 2020).

We observe that our results successfully replicated known results concerning the relationship between personality traits and different mental health indicators (Widiger \& Trull, 1992; Widiger \& Costa, 1994, Mineka, Watson, \& Clark, 1998; Kotov, Gamez, Schmidt, \& Watson, 2010; Beck,
Davis, \& Freeman, 2015). Figure 1C confirms the positive correlation between BIEPS, RS, and PANAS positive affect scores, and negative correlations between STAI trait and state, and PANAS negative affect ( $\mathrm{Hu}$, Zhang, \& Wang, 2015). Concerning personality traits, neuroticism correlated both with temporary psychological states (i.e., negative affect, state anxiety) and more stable personality characteristics (i.e., trait anxiety), confirming a significant association between this trait and negative emotion (Costa and McCrae, 1977, 1980; John, Naumann, \& Soto, 2008). Higher order traits also behaved as expected, beta factor being correlated with the well-being and resilience scores, and alpha factor behaving opposite to the neuroticism trait, i.e., exhibiting similar correlations of the opposite sign (Bäckström, 2007; Kardum \& Hudek-Knezevic, 2012).

We investigated the relationship between self-reported drug use and the psychometric questionnaire scores by first applying principal component analysis to group subjects according to their experiences with psychoactive drugs. These groups reflected how the use of drugs was clustered in our sample: the first component included mainstream psychedelic, entactogen, and dissociative drugs, most of which are consumed in recreational contexts; the second group only included serotonergic psychedelics that are commonly consumed in religious or ceremonial context; the final group included prescription psychoactive drugs such as antidepressants, antipsychotics and sedatives. This classification was useful to highlight the specific effects of certain psychedelic drugs, which likely transcend their pharmacological action and emerge as a consequence of interactions with contextual factors (i.e., set and setting) (Carhart-Harris \& 
Goodwin, 2017; Kazdin, 2007; Mulder, Murray, \& Rucklidge, 2017).

We confirmed that experience with psychedelic drugs was associated with changes in personality traits indexing the experience of novelty (Bouso et al., 2018; Erritzoe et al., 2018, 2019; Lebedev et al., 2016; MacLean et al., 2011). Openness refers to active curiosity in the intellectual domain, while extraversion, represents openness behavioral counterpart but oriented to the material world, involving an active type of curiosity that includes (but is not limited to) social interactions (DeYoung, Peterson, \& Higgins, 2002). Both openness and extraversion are functional characteristics, since a person who faces the environment in a positive way is more likely to obtain reinforcement from the interaction and thus adapt his or her behavior accordingly, suggesting a link between these traits and serotonergic and dopaminergic circuits (Ashby \& Isen, 1999; Depue \& Collins, 1999; DeYoung et al., 2002). As shown in Fig. 1D, the reported number of psychedelic drug uses was positively correlated with openness and extraversion; furthermore, lifetime use of psychedelic were linked to significant increases in these traits (with the exception of the extraversion trait for the "entheogen" group). Although these results are particularly interesting, due to the nature of our study we cannot confirm whether this increment was caused by lifetime use of psychedelic drugs, or due to different preexisting personality traits psychedelic drug users.

Concerning second order personality traits, alpha and beta factors presented significant increases in the groups of psychedelic users, and the reported number of psychedelic uses correlated with both traits. The alpha factor is obtained as a combination of agreeableness, conscientiousness and the inverse of neuroticism, and has been interpreted as a measure of social desirable traits (Bäckström, 2007; Kardum \& Hudek-Knezevic, 2012). This factor has significantly increased in the "recreational" group but not in the "entheogen" group. The beta factor is obtained as a combination of extraversion and openness and has been interpreted as a striving for self-assertion and self-expansion (Bäckström, 2007; Kardum \& Hudek-Knezevic, 2012). This factor was significantly increased in both the "recreational" and "entheogen" group, and also correlated positively with the reported number of psychedelic drug uses. Alternatively, the alpha and beta factors have been interpreted in terms of "stability" and "plasticity", respectively (DeYoung et al., 2002; Digman, 1997). The positive association between lifetime use of psychedelics and these personality traits suggests enhanced resilience and well-being in the light of challenging situations (Fig. 1C).

Certain considerations can be drawn in regards to the link between psychedelics and changes in personality traits. First, since some of these changes could be related to $5-\mathrm{HT}_{2 \mathrm{~A}}$ receptor expression and activation (Kalbitzer et al., 2009), it is hypothesized that a pharmacological interaction occurring in a sustained way could modify genetic expression and thus promote stable modifications in the personality of the users and therefore also in their behavioral patterns (Bouso et al., 2018). In this case, a causal link could exist between the changes in personality trait and the reported number of psychedelic drug uses (Fig. 5C). Second, this could also offer psychotherapeutic potential by external modulation of personality traits. This can be considered both an end by itself (e.g., in the case of certain disorders), or an intermediate objective if the therapeutic goal is to make the patient more flexible to work on psychopathological aspects underlying their personality style (e.g., mood disorders like depression, caused by personality disorders like avoidant personality disorder).

Additionally, all the personality traits evaluated in this study (i.e., extraversion, agreableness, conscientiousness, neuroticism, openness), and their second order factors (i.e., alpha and beta) are psychological representations whose utility is to model the most stable and durable cognitive style of individuals. As a stable factor, personality bears a large part of the variance of the temporal states of mind (e.g., the clinical association between psychopathological conditions and the neuroticism trait). Likewise, these temporary states are caused by the cognitive evaluation resulting from the interaction with the environment, and influence secondary constructions that involve self-evaluation (i.e., well-being and resilience). It is therefore appropriate to propose a logical order in which the variables of this study are linked: the most stable factors (personality traits and anxiety as a trait) influence the cognitive style that is responsible for interpreting and processing reality. In turn, what is interpreted affects emotions, which can be considered as temporary cognitive states (anxiety, positive affect, negative affect). The sustained summation of these states makes up, among other things, the selfperceived processes of well-being and resilience.

We must clarify certain limitations arising from methodology. First, it is not possible to corroborate the information given by the participants, especially concerning the identity and dose of the psychoactive drugs they consumed. Second, it is possible that variables outside the scope of our survey are influencing the results we obtained. Concerning this limitation, our principal component analysis aimed to alleviate the effect of confounds caused by poly-drug use by dividing the sample into disjoint groups depending on their principal component values. Finally, it was not possible to draw causal inferences due to our study design.

In summary, we performed a survey to investigate the relationship between mental health, personality and past drug use during the COVID-19 pandemic. Our results do not suggest an association between past psychedelic use and impaired mental health indicators; on the contrary, we found evidence supporting a more resilient and stable personality structure in those subjects who reported repeated use of certain psychedelic compounds. This study adds to the existing literature on the relationship between mental health and lifetime psychedelic use by investigating self-reported measures of well-being during a highly challenging situation known to cause adverse psychological responses. Future studies should investigate with more detail whether our results can be attributed to the long-term changes induced by psychedelics, and how these changes relate to the available evidence concerning the potential use of psychedelics in the treatment of psychiatric disorders. 
Competing interest statement: The authors declare no competing interest.

\section{ACKNOWLEDGMENTS}

This study has been funded by grant COVID-19 IP 263 by Agencia Nacional de Promoción de la Investigación, el Desarrollo Tecnológico y la Innovación.

\section{APPENDIX A: SUPPLEMENTARY MATERIAL}

Supplementary Material Supplementary data to this article can be found online at https://doi.org/10.1556/2054.2021.00172.

\section{REFERENCES}

Ashby, F. G., \& Isen, A. M. (1999). A neuropsychological theory of positive affect and its influence on cognition. Psychological Review, 106(3), 529.

Bäckström, M. (2007). Higher-order factors in a five-factor personality inventory and its relation to social desirability. European Journal of Psychological Assessment, 23(2), 63-70.

Beck, A. T., Davis, D. D., \& Freeman, A. (Eds.). (2015). Cognitive therapy of personality disorders. Guilford Publications.

Benet-Martinez, V., \& John, O. E. (1998). Los Cinco Grandes Across Cultures and Ethnic Groups: Multitrait Multimethod Analyses of the Big Five in Spanish and English (Vol. 75). Psychological Association, Inc.

Bogenschutz, M. P., Forcehimes, A. A., Pommy, J. A., Wilcox, C. E., Barbosa, P., \& Strassman, R. J. (2015). Psilocybin-assisted treatment for alcohol dependence: A proof-of-concept study. Journal of Psychopharmacology, 29(3), 289-299. https://doi.org/ 10.1177/0269881114565144.

Bouso, J. C., dos Santos, R. G., Alcázar-Córcoles, M. Á., \& Hallak, J. E. C. (2018). Serotonergic psychedelics and personality: A systematic review of contemporary research. Neuroscience \& Biobehavioral Reviews, 87, 118-132. https://doi.org/10.1016/j. neubiorev.2018.02.004.

Brooks, S. K., Webster, R. K., Smith, L. E., Woodland, L., Wessely, S., Greenberg, N., et al. (2020). The psychological impact of quarantine and how to reduce it: Rapid review of the evidence. Lancet, 395(10227), 912-920.

Carhart-Harris, R. L., \& Goodwin, G. M. (2017). The therapeutic potential of psychedelic drugs: Past, present, and future. Neuropsychopharmacology, 42(11), 2105-2113. https://doi.org/10. 1038/npp.2017.84.

Casullo, M., \& Brenlla, M. (2002). Evaluación del bienestar psicológico en Iberoamérica. In Evaluación del bienestar psicológico en Iberoamérica (5). Argentina: Paidós.

Costa, P., Jr., \& McCrae, R. (1977). Psychiatric symptom dimensions in the Cornell Medical Index among normal adult males. Journal of Clinical Psychology, 33(4), 941-946.

Costa, P. T., \& McCrae, R. R. (1980). Influence of extraversion and neuroticism on subjective well-being: Happy and unhappy people. Journal of Personality and Social Psychology, 38(4), 668678. https://doi.org/10.1037/0022-3514.38.4.668.

Depue, R. A., \& Collins, P. F. (1999). Neurobiology of the structure of personality: Dopamine, facilitation of incentive motivation, and extraversion. The Behavioral and Brain Sciences, 22(3), 491-517.

DeYoung, C. G., Peterson, J. B., \& Higgins, D. M. (2002). Higherorder factors of the Big Five predict conformity: are there neuroses of health? Personality and Individual Differences, 33(4), 533-552. https://doi.org/10.1016/S0191-8869(01)00171-4.

Digman, J. (1997). Higher-order factors of the big five. Journal of Personality and Social Psychology, 73(6).

Dyck, E., \& Farrell, P. (2018). Psychedelics and psychotherapy in Canada: Humphry Osmond and Aldous Huxley. History of Psychology, 21(3), 240.

Erritzoe, D., Roseman, L., Nour, M. M., MacLean, K., Kaelen, M., Nutt, D. J., et al. (2018). Effects of psilocybin therapy on personality structure. Acta Psychiatrica Scandinavica, 138(5), 368-378.

Erritzoe, D., Smith, J., Fisher, P. M., Carhart-Harris, R., Frokjaer, V. G., \& Knudsen, G. M. (2019). Recreational use of psychedelics is associated with elevated personality trait openness: Exploration of associations with brain serotonin markers. Journal of Psychopharmacology, 33(9), 1068-1075.

Escohotado, A. (1999). A brief history of drugs: From the stone age to the stoned age.

Gallagher, M. W., Zvolensky, M. J., Long, L. J., Rogers, A. H., \& Garey, L. (2020). The impact of covid-19 experiences and associated stress on anxiety, depression, and functional impairment in American adults. Cognitive Therapy and Research, 44(6), 1043-1051.

Garcia-Romeu, A., Griffiths, R. R., \& Johnson, M. W. (2014). Psilocybin-occasioned mystical experiences in the treatment of tobacco addiction. Current Drug Abuse Reviews, 7(3), 157-164.

Griffiths, R. R., Johnson, M. W., Richards, W. A., Richards, B. D., Jesse, R., MacLean, K. A., et al. (2018). Psilocybin-occasioned mystical-type experience in combination with meditation and other spiritual practices produces enduring positive changes in psychological functioning and in trait measures of prosocial attitudes and behaviors. Journal of Psychopharmacology, 32(1), 49-69.

Hu, T., Zhang, D., \& Wang, J. (2015). A meta-analysis of the trait resilience and mental health. Personality and Individual Differences, 76, 18-27. https://doi.org/10.1016/j.paid.2014.11.039.

Johansen, P. Ø., \& Krebs, T. S. (2015). Psychedelics not linked to mental health problems or suicidal behavior: A population study. Journal of Psychopharmacology, 29(3), 270-279.

John, O. P., Naumann, L. P., \& Soto, C. J. (2008). Paradigm shift to the integrative Big Five Trait taxonomy: History, measurement, and conceptual issues. Handbook of Personality: Theory and Research, 114-158. https://doi.org/10.1016/S0191-8869(97) 81000-8.

Johnson, M. W., Garcia-Romeu, A., Cosimano, M. P., \& Griffiths, R. R. (2014). R agonist psilocybin in the treatment of tobacco addiction 2A pilot study of the 5-HT on behalf of: British association for psychopharmacology can be found at: Journal of psychopharmacology Additional services and information for. Downloaded from Journal of Psychopharmacology, 12(10), 983992. https://doi.org/10.1177/0269881114548296. 
Johnson, M. W., Garcia-Romeu, A., \& Griffiths, R. R. (2017). Longterm follow-up of psilocybin-facilitated smoking cessation. The American Journal of Drug and Alcohol Abuse, 43(1), 55-60. https://doi.org/10.3109/00952990.2016.1170135.

Johnson, M. W., \& Griffiths, R. R. (2017). Potential therapeutic effects of psilocybin. Neurotherapeutics, 14(3), 734-740. https:// doi.org/10.1007/s13311-017-0542-y.

Johnson, M. W., Hendricks, P. S., Barrett, F. S., \& Griffiths, R. R. (2019). Classic psychedelics: an integrative review of epidemiology, therapeutics, mystical experience, and brain network function. Pharmacology \& Therapeutics, 197, 83-102. https://doi. org/10.1016/j.pharmthera.2018.11.010.

Jungaberle, H., Thal, S., Zeuch, A., Rougemont-Bücking, A., von Heyden, M., Aicher, H., et al. (2018). Positive psychology in the investigation of psychedelics and entactogens: a critical review. Neuropharmacology, 142, 179-199. https://doi.org/10.1016/j. neuropharm.2018.06.034.

Kalbitzer, J., Frokjaer, V. G., Erritzoe, D., Svarer, C., Cumming, P., Nielsen, F. A., et al. (2009). The personality trait openness is related to cerebral 5-HTT levels. NeuroImage, 45(2), 280-285.

Kardum, I., \& Hudek-Knezevic, J. (2012). Relationships between five-factor personality traits and specific health-related personality dimensions. International Journal of Clinical and Health Psychology, 12(3), 373-387.

Kazdin, A. E. (2007). Mediators and mechanisms of change in psychotherapy research. Annual Review of Clinical Psychology, 3, 1-27.

Kleber, H. D. (2016). The successful return of psychedelics to psychiatry. Journal of Psychopharmacology, 30(12), 1211.

Kotov, R., Gamez, W., Schmidt, F., \& Watson, D. (2010). Linking "big" personality traits to anxiety, depressive, and substance use disorders: A meta-analysis. Psychological Bulletin, 136(5), 768.

Krebs, T. S., \& Johansen, P. Ø. (2013). Psychedelics and mental health: A population study. Plos One, 8(8), e63972.

Lebedev, A. V., Kaelen, M., Lövdén, M., Nilsson, J., Feilding, A., Nutt, D. J., et al. (2016). LSD-induced entropic brain activity predicts subsequent personality change. Human Brain Mapping, 37(9), 3203-3213. https://doi.org/10.1002/hbm.23234.

Lee, M. A., \& Shlain, B. (1992). Acid dreams: The complete social history of LSD: The CIA, the sixties, and beyond. Grove Press.

MacLean, K. A., Johnson, M. W., \& Griffiths, R. R. (2011). Mystical experiences occasioned by the hallucinogen psilocybin lead to increases in the personality domain of openness. Journal of Psychopharmacology, 25(11), 1453-1461. https://doi.org/10. 1177/0269881111420188.

Mineka, S., Watson, D., \& Clark, L. A. (1998). Comorbidity of anxiety and unipolar mood disorders. Annual Review of Psychology, 49(1), 377-412.

Mulder, R., Murray, G., \& Rucklidge, J. (2017). Common versus specific factors in psychotherapy: Opening the black box. The Lancet Psychiatry, 4(12), 953-962.

Nichols, D. E. (2016). Psychedelics. Pharmacological Reviews, 68(2), 264-355. https://doi.org/10.1124/pr.115.011478.

Nichols, C. D., \& Hendricks, P. S. (2020). Classic psychedelics as therapeutics for psychiatric disorders. In Handbook of
Behavioral Neuroscience (31, pp. 959-966). Elsevier. https://doi. org/10.1016/B978-0-444-64125-0.00049-9.

Nutt, D., \& Carhart-Harris, R. (2020). The current status of psychedelics in psychiatry. JAMA Psychiatry, 78(2), 121-122.

Nutt, D. J., King, L. A., \& Phillips, L. D. (2010). Drug harms in the UK: A multicriteria decision analysis. Lancet, 376(9752), 15581565.

Palamar, J. J., \& Acosta, P. (2020). A qualitative descriptive analysis of effects of psychedelic phenethylamines and tryptamines. Human Psychopharmacology: Clinical and Experimental, 35(1), e2719.

Pfefferbaum, B., \& North, C. S. (2020). Mental health and the Covid-19 pandemic. New England Journal of Medicine, 383(6), 510-512.

Preller, K. H., \& Vollenweider, F. X. (2016). Phenomenology, structure, and dynamic of psychedelic states. In Behavioral neurobiology of psychedelic drugs (pp. 221-256). Berlin, Heidelberg: Springer.

Rätsch, C. (2005). The encyclopedia of psychoactive plants: Ethnopharmacology and its applications. Simon and Schuster.

Reiff, C. M., Richman, E. E., Nemeroff, C. B., Carpenter, L. L., Widge, A. S., Rodriguez, C. I., et al.et al. (2020). Psychedelics and psychedelic-assisted psychotherapy. American Journal of Psychiatry, 177(5), 391-410.

Rodríguez, M., Pereyra, M. G., Gil, E., Jofré, M., Bortoli, M. De, \& Labiano, L. M. (2009). Propiedades psicométricas de la escala de resiliencia versión argentina. Revista Evaluar, 9(0), 72-82. https://doi.org/10.35670/1667-4545.v9.n1.465.

Rucker, J. J., Iliff, J., \& Nutt, D. J. (2018). Psychiatry \& the psychedelic drugs. Past, present \& future. Neuropharmacology, 142, 200-218.

Schmid, Y., Enzler, F., Gasser, P., Grouzmann, E., Preller, K. H., Vollenweider, F. X., et al. (2015). Acute effects of lysergic acid diethylamide in healthy subjects. Biological Psychiatry, 78(8), 544-553.

Spielberger, C., Gorsuch, R., Lushene, R., \& Inc, P. V. (1983). Manual for the state-trait anxiety inventory. Palo Alto, CA: Consulting Psychologists Press.

Wagnild, G. M., \& Young, H. M. (1993). Development and psychometric. Journal of Nursing Measurement, 1(2), 165-17847.

Watson, D., Clark, L., \& Tellegen, A. (1988). Development and validation of brief measures of positive and negative affect: The PANAS scales. Journal of Personality and Social Psychology, 54(6), 1063-1070. Retrieved from https:// psycnet.apa.org/journals/psp/54/6/1063.html?uid=198831508-001.

Widiger, T. A., \& Costa, Jr, P. T. (1994). Personality and personality disorders. Journal of Abnormal Psychology, 103(1), 78.

Widiger, T. A., \& Trull, T. J. (1992). Personality and psychopathology: An application of the five-factor model. Journal of Personality, 60(2), 363-393.

Zacher, H., \& Rudolph, C. W. (2020). Individual differences and changes in subjective wellbeing during the early stages of the COVID-19 pandemic. American Psychologist, 76(1), 50-62.

Open Access. This is an open-access article distributed under the terms of the Creative Commons Attribution-NonCommercial 4.0 International License (https:// creativecommons.org/licenses/by-nc/4.0/), which permits unrestricted use, distribution, and reproduction in any medium for non-commercial purposes, provided the original author and source are credited, a link to the CC License is provided, and changes - if any - are indicated. 